\title{
Recent developments in heavy-ion fusion reactions around the Coulomb barrier
}

\author{
K. Hagino ${ }^{1,2,3}$, N. Rowley ${ }^{4}$, and J.M. Yao ${ }^{1,5,6}$ \\ ${ }^{1}$ Department of Physics, Tohoku University, Sendai 980-8578, Japan \\ ${ }^{2}$ Research Center for Electron Photon Science, Tohoku University, 1-2-1 Mikamine, Sendai 982-0826, Japan \\ ${ }^{3}$ National Astronomical Observatory of Japan, 2-21-1 Osawa, Mitaka, Tokyo 181-8588, Japan \\ ${ }^{4}$ Institut de Physique Nucléaire, UMR 8608, CNRS-IN2P3 et Université de Paris Sud, 91406 Orsay Cedex, \\ France \\ ${ }^{5}$ School of Physical Science and Technology, Southwest University, Chongqing 400715, China \\ ${ }^{6}$ Department of Physics and Astronomy, University of North Carolina, Chapel Hill, North Carolina 27516- \\ 3255, USA
}

\begin{abstract}
The nuclear fusion is a reaction to form a compound nucleus. It plays an important role in several circumstances in nuclear physics as well as in nuclear astrophysics, such as synthesis of superheavy elements and nucleosynthesis in stars. Here we discuss two recent theoretical developments in heavy-ion fusion reactions at energies around the Coulomb barrier. The first topic is a generalization of the Wong formula for fusion cross sections in a single-channel problem. By introducing an energy dependence to the barrier parameters, we show that the generalized formula leads to results practically indistinguishable from a full quantal calculation, even for light symmetric systems such as ${ }^{12} \mathrm{C}+{ }^{12} \mathrm{C}$, for which fusion cross sections show an oscillatory behavior. We then discuss a semi-microscopic modeling of heavy-ion fusion reactions, which combine the coupled-channels approach to the state-of-the-art nuclear structure calculations for low-lying collective motions. We apply this method to subbarrier fusion reactions of ${ }^{58} \mathrm{Ni}+{ }^{58} \mathrm{Ni}$ and ${ }^{40} \mathrm{Ca}+{ }^{58} \mathrm{Ni}$ systems, and discuss the role of anharmonicity of the low-lying vibrational motions.
\end{abstract}

\section{Introduction}

Fusion is defined as a reaction in which two separate nuclei combine together to form a (hot) compound nucleus. It is indispensable to understand its reaction dynamics in order to understand synthesis of superheavy elements and nucleosynthesis in stars. Moreover, fusion reactions inherently contain rich physics, as there is a strong interplay in it, especially at energies around the Coulomb barrier, between the nuclear reaction and the nuclear structure. That is, it has been known well that fusion cross sections at subbarrier energies are largely enhanced relative to a prediction of a one-dimensional potential model, due to the couplings of the relative motion between the colliding nuclei to several nuclear intrinsic motions [1-5]. This is in marked contrast to high energy nuclear reactions, in which the reaction dynamics is much simpler and the distorted wave Born approximation (DWBA) often suffices its treatment. Fusion also offers a unique opportunity to study quantum tunneling with many degrees 
of freedom. That is, fusion takes place only by quantum tunneling at energies below the Coulomb barrier, and the subbarrier enhancement of fusion cross sections can be viewed as a consequence of a coupling assisted tunneling. Heavy-ion fusion reactions are unique in this respect because a variety of intrinsic degrees of freedom are involved, such as a surface vibration with several multipolarities, various sort of nuclear deformations and the associated rotational motion, and several types of particle transfer processes. This is in contrast to atomic and molecular collisions, in which only a limited types of intrinsic motion are involved. Also, in heavy-ion fusion reactions, the incident energy can be varied in order to study the energy dependence of the tunneling probability, whereas the energy is fixed in many other tunneling phenomena in nuclear physics, such as alpha decays.

In this contribution, we shall discuss two recent theoretical developments in heavy-ion fusion reactions. The first topic is a generalization of the Wong formula for fusion cross sections [6]. Although this celebrated formula yields almost exact results for single-channel calculations for relatively heavy systems such as ${ }^{16} \mathrm{O}+{ }^{144} \mathrm{Sm}$, it tends to overestimate the cross section for light systems such as ${ }^{12} \mathrm{C}+{ }^{12} \mathrm{C}$. We generalize the formula to take account of the energy dependence of the barrier parameters and show that the energy-dependent version reproduces almost perfectly results of a full quantal calculation. We also discuss the deviations arising from the discrete nature of the intervening angular momenta, whose effect can lead to an oscillatory contribution to the excitation function.

The second topic is a semi-microscopic modeling of heavy-ion fusion reactions [7]. For this purpose, we first describe microscopically low-lying collective excitations of atomic nuclei with the multi-reference covariant density functional theory, and combine them with coupled-channels calculations. We use the calculated transition strengths among several collective states as inputs to the coupled-channels calculations. This approach provides a natural way to describe anharmonic multiphonon excitations as well as a deviation of rotational excitations from a simple rigid rotor. We apply this method to subbarrier fusion reactions of ${ }^{58} \mathrm{Ni}+{ }^{58} \mathrm{Ni}$ and ${ }^{40} \mathrm{Ca}+{ }^{58} \mathrm{Ni}$ systems, and discuss the role of anharmonicity in subbarrier fusion of these systems.

\section{Generalization of the Wong formula for fusion cross sections}

Let us first discuss the well-known Wong formula for fusion cross sections. The simplest approach to heavy-ion fusion reactions is to use the potential model, assuming that both the projectile and the target nuclei are inert. Fusion cross sections are then obtained by calculating the $S$-matrix, $S_{l}$, for each partial wave. If all the flux crossing the Coulomb barrier fuses, the fusion cross sections $\sigma_{\text {fus }}$ read

$$
\sigma_{\text {fus }}(E)=\frac{\pi}{k^{2}} \sum_{l=0}^{\infty}(2 l+1) P_{l}(E),
$$

where $k=\sqrt{2 \mu E / \hbar^{2}}$ is the wave number associated with the energy $E$ ( $\mu$ being the reduced mass) and $P_{l}(E)=1-\left|S_{l}\right|^{2}$ is the penetrability of the Coulomb barrier for the $l$-th partial wave.

Wong has derived a compact approximate expression for Eq. (1) [8], which has by now been known as the Wong formula. To this end, Wong introduced the following three approximations:

- the parabolic approximation. The total (that is, the nuclear + the Coulomb) inter-nucleus potential $V_{0}(r)$ for the $s$-wave is approximated by a parabolic function, that is,

$$
V_{0}(r) \sim V_{b}-\frac{1}{2} \mu \Omega^{2}\left(r-R_{b}\right)^{2}
$$

where $V_{b}, R_{b}$, and $\hbar \Omega$ are the barrier height, the barrier position, and the "barrier curvature", respectively. 

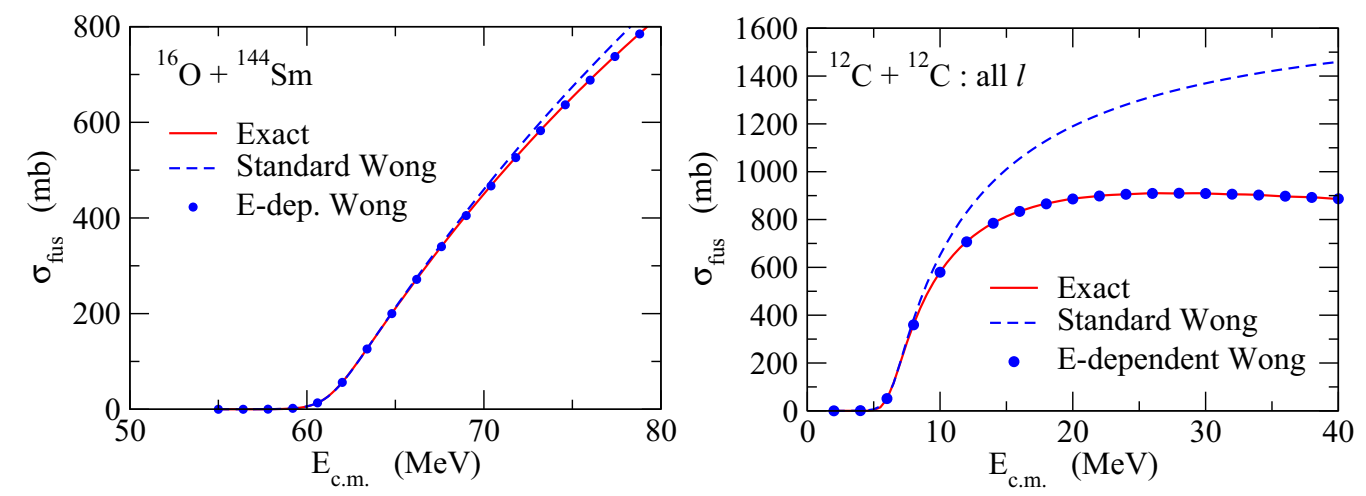

Figure 1. Fusion cross sections for the ${ }^{16} \mathrm{O}+{ }^{144} \mathrm{Sm}$ (the left panel) and the ${ }^{12} \mathrm{C}+{ }^{12} \mathrm{C}$ (the right panel) systems. The solid lines show the results of a full quantal calculation, while the dashed line shows the fusion cross sections evaluated with the Wong formula, Eq. (6). The filled circles, on the other hand, denote the results of the generalized (the energy dependent) Wong formula given by Eq. (9). The Bose symmetry of the identical spin- 0 system is ignored for the ${ }^{12} \mathrm{C}+{ }^{12} \mathrm{C}$ system, and both even and odd partial waves are summed up in the cross sections.

- the l-independent barrier position and curvature. The barrier position, $R_{b}$, and the barrier curvature, $\hbar \Omega$, are assumed to be independent of $l$. In this case, the effective potential for the $l$-th partial wave reads,

$$
V_{0}(r)+\frac{l(l+1) \hbar^{2}}{2 \mu r^{2}} \sim V_{b}+\frac{l(l+1) \hbar^{2}}{2 \mu R_{b}^{2}}-\frac{1}{2} \mu \Omega^{2}\left(r-R_{b}\right)^{2} .
$$

The penetrability can then be calculated analytically with the Hill-Wheeler formula [9] as,

$$
P_{l}(E)=\frac{1}{1+\exp \left[\frac{2 \pi}{\hbar \Omega}\left(V_{b}+\frac{l(l+1) \hbar^{2}}{2 \mu R_{b}^{2}}-E\right)\right]}
$$

- the continuum approximation for $l$. The angular momentum $l$ is treated as a continuous variable and the sum in Eq. (1) is replaced by the integral,

$$
\sigma_{\text {fus }}(E)=\frac{\pi}{k^{2}} \sum_{l=0}^{\infty}(2 l+1) P_{l}(E) \rightarrow \frac{\pi}{k^{2}} \int_{0}^{\infty} d l(2 l+1) P_{l}(E) .
$$

With these approximations, the fusion cross sections are obtained as $[1,3,8]$,

$$
\sigma_{\text {fus }}(E)=\frac{\hbar \Omega}{2 E} R_{b}^{2} \ln \left[1+\exp \left(\frac{2 \pi}{\hbar \Omega}\left(E-V_{b}\right)\right)\right] .
$$

Notice that this formula yields the classical fusion cross sections,

$$
\sigma_{\text {fus }}(E) \sim \pi R_{b}^{2}\left(1-\frac{V_{b}}{E}\right)
$$

at energies well above the Coulomb barrier, $E-V_{b} \gg \hbar \Omega / 2 \pi$. 
The left panel of Fig. 1 shows the performance of the Wong formula for the ${ }^{16} \mathrm{O}+{ }^{144} \mathrm{Sm}$ system. The solid line is obtained quantum mechanically by solving the Schrödinger equation for a given internucleus potential, while the dashed line is obtained with the Wong formula, Eq. (6). Even though the Wong formula slightly overestimates the fusion cross sections at energies well above the Coulomb barrier, reflecting the fact that the barrier position gradually decreases as a function of $l$, the overall agreement is satisfactory. In contrast, the situation drastically changes for light systems. The right panel of Fig. 1 shows a comparison between the quantal and the approximate fusion cross sections for the ${ }^{12} \mathrm{C}+{ }^{12} \mathrm{C}$ system. Here, in order to simplify the discussion, for the moment we ignore the Bose symmetry of this identical spin-0 system and sum over all even and odd partial waves. One can see that the Wong formula largely overestimates fusion cross sections, and the agreement is much worse than the heavier system, ${ }^{16} \mathrm{O}+{ }^{144} \mathrm{Sm}$. This is because the centrifugal potential plays a more important role in light systems, and the Coulomb barrier is not rigid against a variation of angular momentum [6].

One can improve the Wong formula by introducing the energy dependence to the barrier parameters $\left[V_{b}, R_{b}, \hbar \Omega\right]$. To this end, we first introduce the grazing angular momentum $l_{g}$, at which the barrier height of the effective potential becomes identical to the incident energy, $E$. If we denote the barrier position for $l_{g}$ as $R_{E}, l_{g}$ and $R_{E}$ are related to each other as,

$$
\frac{l_{g}\left(l_{g}+1\right) \hbar^{2}}{2 \mu R_{E}^{2}}=E-V_{E},
$$

where $V_{E}=V_{0}\left(R_{E}\right)$ is the sum of the Coulomb and the nuclear potentials at $R_{E}$. An idea of the generalized Wong formula is to replace the barrier parameters $\left[V_{b}, R_{b}, \hbar \Omega\right]$ with the energy dependent ones, $\left[V_{E}, R_{E}, \hbar \Omega_{E}\right]$, where $\hbar \Omega_{E}$ is the barrier curvature for the grazing angular momentum $l_{g}$. That is, the generalized version of the Wong formula now reads [6],

$$
\sigma_{\text {fus }}(E)=\frac{\hbar \Omega_{E}}{2 E} R_{E}^{2} \ln \left[1+\exp \left(\frac{2 \pi}{\hbar \Omega_{E}}\left(E-V_{E}\right)\right)\right] .
$$

Notice that we still assume an $l$-independent barrier position and curvature in integrating Eq. (5). However, the generalization is such that these parameters are evaluated at the grazing angular momentum for each energy $E$, rather than using the values for the $s$-wave. The filled circles in Fig. 1 show the fusion cross sections obtained with the generalized Wong formula, Eq. (9). One can see that the quantal results are well reproduced by introducing the energy dependence to the barrier parameters, both for the ${ }^{16} \mathrm{O}+{ }^{144} \mathrm{Sm}$ and the ${ }^{12} \mathrm{C}+{ }^{12} \mathrm{C}$ systems.

One can also go beyond the continuum approximation for the angular momentum sum. Using the Poisson sum formula [10], the next order correction to the Wong formula reads [6, 11],

$$
\sigma_{\text {fus }}(E) \sim \sigma_{\text {Wong }}+\sigma_{\text {osc }},
$$

where $\sigma_{\text {Wong }}$ is the smooth part of fusion cross sections given by the Wong formula, Eq. (9), while the oscillatory contribution $\sigma_{\text {osc }}$ is given by $[6,11]$,

$$
\sigma_{\mathrm{osc}}=2 \pi R_{E}^{2} \frac{\hbar \Omega_{E}}{E} e^{-2 \xi} \sin \left(2 \pi l_{g}\right)
$$

with

$$
\xi=\frac{\hbar \Omega_{E}}{2 l_{g}+1} \cdot \frac{\pi \mu R_{E}^{2}}{\hbar^{2}}
$$




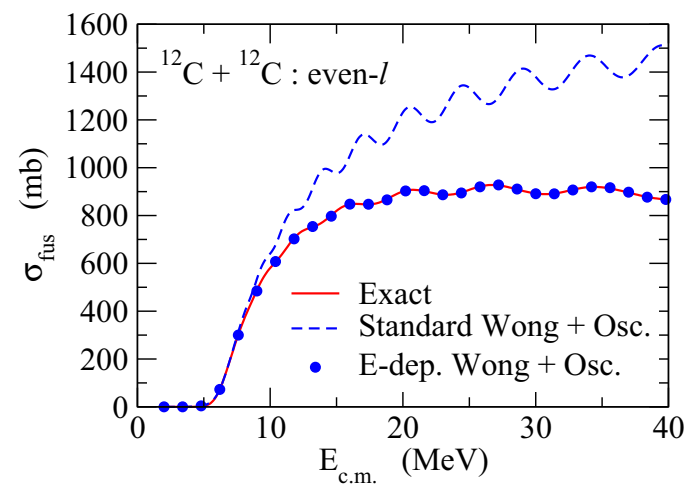

Figure 2. Fusion cross sections for the ${ }^{12} \mathrm{C}+{ }^{12} \mathrm{C}$ system obtained with even partial waves only. The solid line shows the exact quantal result. The filled circles and the dashed line denote the results of the Wong formula supplemented with the oscillatory contribution, Eq. (13), with and without the energy dependence of the barrier parameters, respectively.

Usually, the oscillatory contribution, $\sigma_{\mathrm{osc}}$, is much smaller than the smooth part given by $\sigma_{\text {Wong. }}$. There are, however, certain circumstances where the oscillatory contribution becomes significantly large. This is for a light symmetric system of two identical spin-0 bosons, in which all odd partial waves disappear due to the symmetrization of the wave function. In this case, the fusion cross section is given by twice the sum over the even partial waves. The resultant formula now becomes [6,11],

$$
\sigma_{\mathrm{osc}}=2 \pi R_{E}^{2} \frac{\hbar \Omega_{E}}{E} e^{-\xi} \sin \left(\pi l_{g}\right),
$$

whereas the smooth part is still given by the Wong formula, Eq. (9). The oscillatory part is now significantly larger than before, since the negative exponent has been reduced by a factor of 2 , that is $e^{-2 \xi} \rightarrow e^{-\xi}$.

Figure 2 shows fusion cross sections for the ${ }^{12} \mathrm{C}+{ }^{12} \mathrm{C}$ system, obtained by taking only the even partial waves. The meaning of each line is the same as in Fig. 1. One can see that the fusion cross sections significantly oscillate as a function of $E$. The generalized Wong formula, supplemented with the oscillatory correction, gives an excellent approximation to the exact results.

\section{Semi-microscopic modeling of heavy-ion fusion reactions with a beyond-mean field method}

The potential model presented in the previous section works for light systems, such as ${ }^{14} \mathrm{~N}+{ }^{12} \mathrm{C}$, but it largely underestimates fusion cross sections for heavier systems, such as ${ }^{16} \mathrm{O}+{ }^{154} \mathrm{Sm}$, at energies below the Coulomb barrier [1]. It has been well recognized by now that this large enhancement of subbarrier fusion cross sections is caused by the couplings of the relative motion between the colliding nuclei to several nuclear intrinsic degrees of freedom, such as low-lying collective excitations in the colliding nuclei as well as several nucleon transfer processes [1-4]. A natural framework for heavyion subbarrier fusion has thus been the coupled-channels method with relevant degrees of freedom $[1,12]$. This approach has not only successfully accounted for the subbarrier enhancement of fusion cross sections for many systems but has also provided a natural interpretation of the so called fusion barrier distributions $[1,2,13,14]$. 


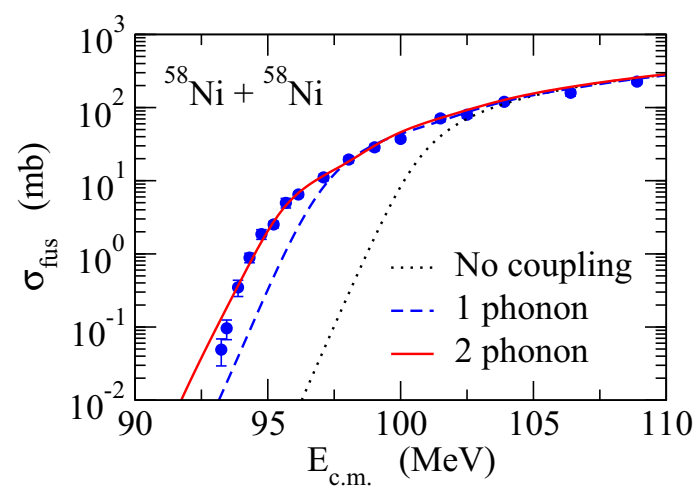

Figure 3. The results of coupled-channels calculations for the fusion cross sections for the ${ }^{58} \mathrm{Ni}+{ }^{58} \mathrm{Ni}$ system. The vibrational coupling to multi-quadrupole-phonon states are considered in the harmonic oscillator approximation. The experimental data are taken from Ref. [15].

In the coupled-channels approach, if the projectile nucleus is assumed to be inert, one expands the total wave function of the system in terms of the eigen-functions of the collective states in the target nucleus, $\left|\varphi_{I 0}\right\rangle$, as

$$
\Psi_{L M_{L}}(\boldsymbol{r})=\sum_{I} \frac{u_{I}(r)}{r} Y_{L M_{L}}(\hat{\boldsymbol{r}})\left|\varphi_{I 0}\right\rangle,
$$

where $I$ and $L$ are the angular momenta for the target state and for the relative motion, respectively. Here, we have introduced the isocentrifugal approximation [1], and have assumed that $L$ and $M_{L}$ (thus also $M_{I}$ ) do not change by the excitation of the target nucleus. Substituting Eq. (14) to the projected Schrödinger equation, $\left\langle\varphi_{I 0}|H-E| \Psi_{L M_{L}}\right\rangle=0$, the coupled-channels equations for the radial wave functions $u_{I}(r) \operatorname{read}[1]$,

$$
\left[-\frac{\hbar^{2}}{2 \mu} \frac{d^{2}}{d r^{2}}+\frac{L(L+1) \hbar^{2}}{2 \mu r^{2}}+V_{0}(r)-E+\epsilon_{I}\right] u_{I}(r)+\sum_{I^{\prime}} V_{I I^{\prime}}(r) u_{I^{\prime}}(r)=0
$$

where $\epsilon_{I}$ is the energy of the target state $I . V_{I I^{\prime}}(r)$ are the coupling matrix elements given by

$$
V_{I I^{\prime}}(r)=\left\langle\varphi_{I 0}\left|V_{\text {coup }}\left(r, \alpha_{\lambda 0}\right)\right| \varphi_{I^{\prime} 0}\right\rangle,
$$

where $V_{\text {coup }}$ is the coupling potential and $\alpha_{\lambda 0}$ is the excitation operator with a multipolarity $\lambda$.

In heavy-ion fusion reactions at energies around the Coulomb barrier, multiple excitations to higher members of collective modes, such as multi-phonon states and high-spin states in the ground state rotational band, often play an important role $[2,14,16]$. As an example, Fig. 3 shows fusion cross sections for the ${ }^{58} \mathrm{Ni}+{ }^{58} \mathrm{Ni}$ system obtained with the coupled-channels calculations which take into account the vibrational coupling to quadrupole phonon states. One can see that the coupling to the first excited state is insufficient in order to account for the subbarrier enhancement of fusion cross sections for this system. In order to take into account the multiple excitations in coupled-channels calculations, one usually uses the simple harmonic oscillator model for vibrational nuclei [1]. In this approximation, the $n$-phonon multiplet states are combined together to form a single effective channel as [1]

$$
|n\rangle=\frac{1}{\sqrt{n !}}\left(a_{\lambda 0}^{\dagger}\right)^{n}|0\rangle
$$




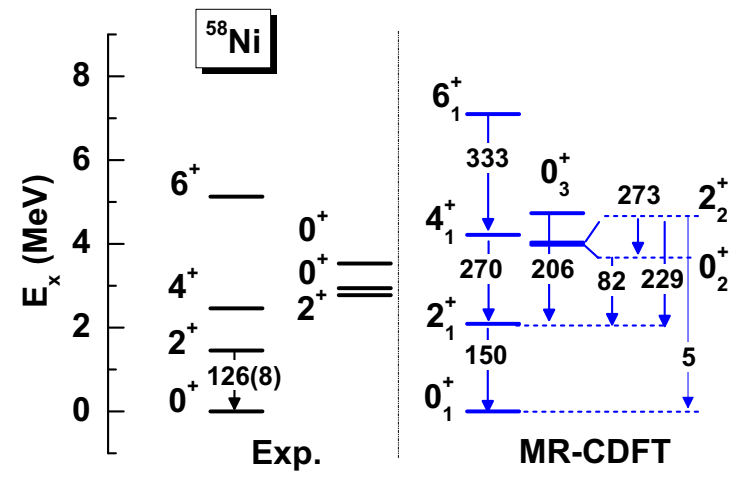

Figure 4. The low-lying energy spectra of ${ }^{58} \mathrm{Ni}$ obtained with the multi-reference covariant density functional theory (MR-CDFT) method with the PC-PK1 force. The arrows indicate the E2 transition strengths, given in units of $e^{2} \mathrm{fm}^{4}$. The experimental data are taken from Refs. [20,21].

where $a_{\lambda 0}^{\dagger}$ is the phonon creation operator and $|0\rangle$ is the vacuum state. The energy of this channel is given by $\epsilon_{n}=n \hbar \omega$, where $\hbar \omega$ is the oscillator quantum, and the matrix elements of $\alpha_{\lambda 0}$ are related to the phonon numbers as $\left\langle n\left|\alpha_{\lambda 0}\right| n^{\prime}\right\rangle \propto \sqrt{n^{\prime}} \delta_{n, n^{\prime}-1}+\sqrt{n^{\prime}+1} \delta_{n, n^{\prime}+1}$. The solid line in Fig. 3 shows the result of the two phonon couplings. One can see that the subbarrier fusion enhancement is now well reproduced.

Even though the harmonic oscillator approximation appears to work well for the ${ }^{58} \mathrm{Ni}+{ }^{58} \mathrm{Ni}$ system, most nuclei, including ${ }^{58} \mathrm{Ni}$, do not have a pure harmonic oscillator spectrum in reality. Concerning the ${ }^{58} \mathrm{Ni}$ nucleus, the degeneracy of the experimentally known two-phonon triplet is considerably broken (see Fig. 4), and also a recent theoretical calculation indicates that the $B(E 2)$ strengths among the collective levels in ${ }^{58} \mathrm{Ni}$ deviate largely from the harmonic oscillator limit [17]. Such anharmonic vibrations can be described, e.g., a multi-reference density-functional theory (MR-DFT), which has been rapidly developed for the past decade $[18,19]$. This method is based on the so called beyondmean-field approximation, which incorporates the angular momentum and particle number projections for a mean-field wave function. The quantum fluctuation of the mean-field wave function is also taken into account with the generator coordinate method (GCM).

Figure 4 shows the spectrum of the ${ }^{58} \mathrm{Ni}$ nucleus [7] constructed with the MR-DFT calculation with the covariant density functional with the PC-PK1 interaction [22]. One can see that the excitation energies of the $0_{2}^{+}, 2_{2}^{+}$, and $4_{1}^{+}$states are about twice the energy of the $2_{1}^{+}$state. On the other hand, it is interesting to notice that the overall pattern of $B(E 2)$ values is quite different from what would be expected for a harmonic vibrator, in which the $B(E 2)$ value from any of the two-phonon triplet states to the $2_{1}^{+}$state is exactly twice the $B(E 2)$ value from the $2_{1}^{+}$state to the ground state. In particular, the $E 2$ transition from the $0_{2}^{+}$to the $2_{1}^{+}$states is much smaller than that for the $4_{1}^{+} \rightarrow 2_{1}^{+}$and the $2_{2}^{+} \rightarrow 2_{1}^{+}$ transitions. Instead, the $0_{2}^{+}$state has a strong transition from the $2_{2}^{+}$state, which clearly indicates that the $0_{2}^{+}$state is not a member of the two-phonon triplet. Compared to the $0_{2}^{+}$state, the $E 2$ transition strength for the $0_{3}^{+} \rightarrow 2_{1}^{+}$transition is much larger and is comparable to that for the $4_{1}^{+} \rightarrow 2_{1}^{+}$and the $2_{2}^{+} \rightarrow 2_{1}^{+}$transitions. This suggests that the $0_{3}^{+}$state is a better candidate for a member of the two-phonon triplets than the $0_{2}^{+}$state, even though the excitation energy is a little large.

In order to take into account such anharmonic nature of the vibrational excitations of ${ }^{58} \mathrm{Ni}$ in the coupled-channels calculations, we first replace the operator $\alpha_{\lambda 0}$ in Eq. (16) with the corresponding 

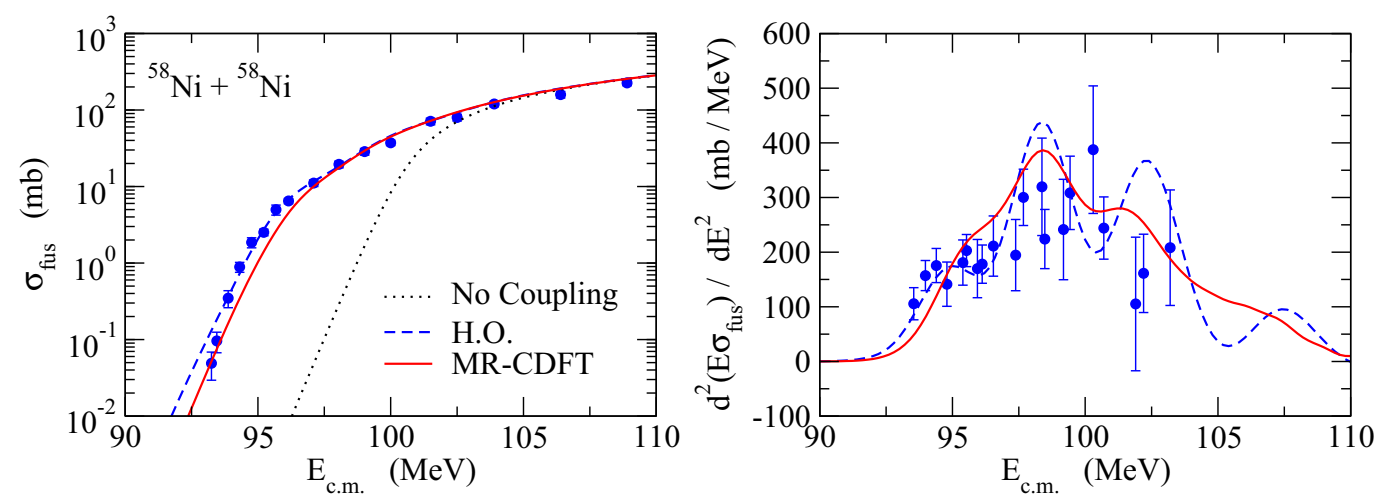

Figure 5. The fusion cross sections (the left panel) and the fusion barrier distributions (the right panel) for the ${ }^{58} \mathrm{Ni}+{ }^{58} \mathrm{Ni}$ system. The dashed line is the result of the coupled-channels calculations including the double quadrupole phonon excitations in each ${ }^{58} \mathrm{Ni}$ nucleus in the harmonic oscillator limit. The solid line, on the other hand, is obtained with the multi-reference covariant density functional theory (MR-CDFT) method by including the couplings to the $0_{1}^{+}, 2_{1}^{+}, 0_{3}^{+}, 2_{2}^{+}$, and $4_{1}^{+}$states in ${ }^{58} \mathrm{Ni}$. The dotted line in the left panel denotes the result in the absence of the channel couplings. The experimental data are taken from Ref. [15] for the fusion cross sections and from Ref. [16] for the fusion barrier distribution.

microscopic multipole operator. That is, we replace the matrix elements of $\alpha_{\lambda 0}$ as,

$$
\left\langle\varphi_{I 0}\left|\alpha_{\lambda 0}\right| \varphi_{I^{\prime} 0}\right\rangle \rightarrow \frac{4 \pi}{3 Z_{T} e R_{T}^{\lambda}}\left\langle\varphi_{I 0}\left|Q_{\lambda 0}\right| \varphi_{I^{\prime} 0}\right\rangle,
$$

where $Q_{\lambda \mu}=\sum_{i} r_{i}^{\lambda} Y_{\lambda \mu}\left(\hat{r}_{i}\right)$ is the microscopic multipole operator, and $R_{T}$ and $Z_{T}$ are the radius and the charge number of the target nucleus, respectively. $\left|\varphi_{I^{\prime} 0}\right\rangle$ on the right hand side is a many-body wave function evaluated with the MR-CDFT method. We still use a phenomenological potential for $V_{0}(r)$ and $V_{\text {coup }}\left(r, \alpha_{\lambda 0}\right)$, and we call our method a semi-microscopic approach.

In heavy-ion fusion reactions, the coupling of the ground state to the lowest-lying collective state is most important. The strength for such coupling can often be estimated from an experimental transition probability. Since it is too much to expect that a MR-CDFT calculation agrees perfectly with experimental data, we introduce an overall scaling factor to the matrix elements, Eq. (18), so that the transition from the lowest-lying collective state to the ground state is consistent with experimental data. The MR-CDFT calculation then provides the relative strengths among collective levels, which are often not available experimentally. The excitation energy, on the other hand, is often known well for many levels, and we simply take them in the calculations whenever they are available experimentally.

Figures 5 shows the fusion cross section $\sigma_{\text {fus }}(E)$ and the fusion barrier distribution $D_{\text {fus }}(E)=$ $d^{2}\left(E \sigma_{\text {fus }}\right) / d E^{2}[2,13]$ for the ${ }^{58} \mathrm{Ni}+{ }^{58} \mathrm{Ni}$ reaction so obtained. The dashed line shows the result of the coupled-channels calculations including up to the double phonon states in the harmonic oscillator limit. All the mutual excitations between the projectile and the target nuclei are included. On the other hand, the solid line in the figure is obtained with the coupling strengths evaluated with the MR-CDFT method. To this end, we include the $0_{1}^{+}, 2_{1}^{+}, 0_{3}^{+}, 2_{2}^{+}$, and $4_{1}^{+}$states in ${ }^{58} \mathrm{Ni}$ in the coupled-channels calculations. Again, all the mutual excitation channels are taken into account. For comparison, the figure also shows the result of no-coupling limit by the dotted line. One can see that the calculations in the harmonic limit overestimate fusion cross sections at the two lowest energies, while the MR- 

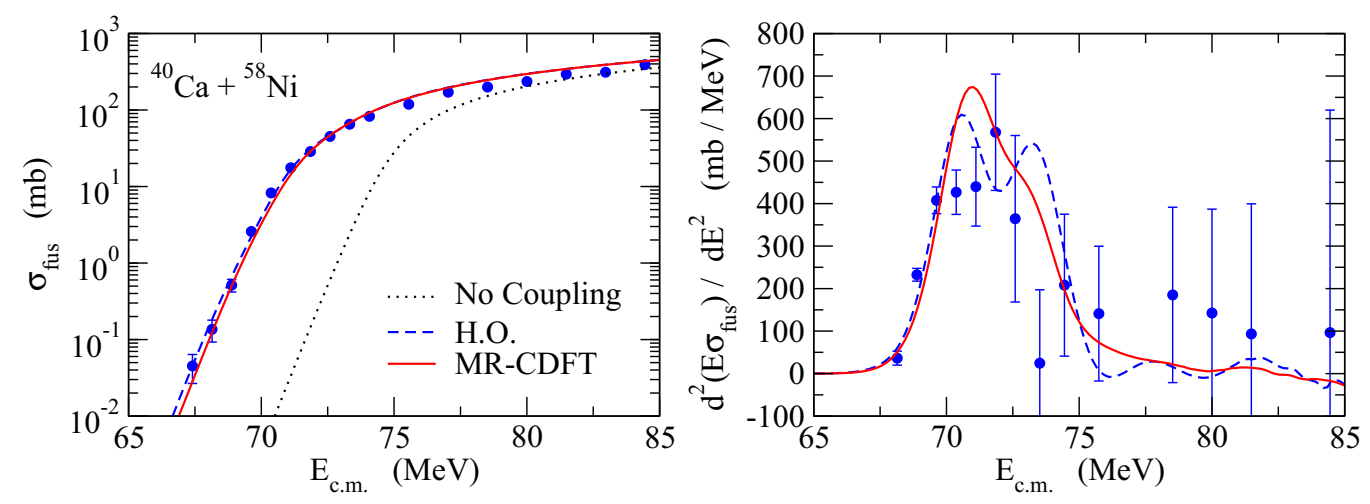

Figure 6. Same as Fig. 5, but for the ${ }^{40} \mathrm{Ca}+{ }^{58} \mathrm{Ni}$ system. The experimental data are taken from Ref. [23].

CDFT calculations underpredict fusion cross sections around $95 \mathrm{MeV}$. The energy dependence of fusion cross sections can be investigated with the fusion barrier distribution, which is plotted in the right panel of the figure. Even though the effect of anharmonicity is not large, one observes that the MR-CDFT calculation leads to a minor improvement by considerably smearing each peak.

We carry out similar calculations also for the ${ }^{40} \mathrm{Ca}+{ }^{58} \mathrm{Ni}$ system [7] (see Fig. 6). The effect of channel coupling is much smaller than in the ${ }^{58} \mathrm{Ni}+{ }^{58} \mathrm{Ni}$ system due to a smaller charge product, $Z_{P} Z_{T}$. Nevertheless, we again observe that the anharmonicity effect in ${ }^{58} \mathrm{Ni}$ smears the fusion barrier distribution, leading to a somewhat better agreement with the experimental fusion barrier distribution as compared to the results in the harmonic oscillator limit.

\section{Summary}

The research field of heavy-ion subbarrier fusion reactions started in the late '70s, when a large enhancement of fusion cross sections was experimentally discovered with respect to the prediction of a simple potential model. The Wong formula has been widely used to estimate fusion cross sections for a given single-channel potential, providing reference cross sections in the absence of channel couplings to discuss the subbarrier enhancement of cross sections. In this contribution, we have first extended the Wong formula by including the energy dependence of the parameters entering the formula, that is, the barrier height, the barrier position, and the barrier curvature. Evaluating these parameters for the grazing angular momentum at each energy, rather than at $l=0$, we have shown that the energy-dependent version of Wong's formula reproduces the exact result well, even for light systems. We have also derived a compact analytic formula for the oscillatory part of fusion cross sections, which originate from the discrete nature of angular momentum. These oscillatory parts become important in light symmetric systems, for which the symmetrization of the system amplifies the oscillations.

We have then proposed a semi-microscopic approach to heavy-ion subbarrier fusion reactions. The basic idea of this approach is to combine the state-of-the-art nuclear structure calculations with coupled-channels calculations. For this purpose, we have used a multi-reference density functional theory (MR-DFT) based on the beyond-mean-field approach. The MR-DFT provides transition strengths among collective states without resorting to the harmonic oscillator model. We have applied this approach to the ${ }^{58} \mathrm{Ni}+{ }^{58} \mathrm{Ni}$ and ${ }^{40} \mathrm{Ca}+{ }^{58} \mathrm{Ni}$ fusion reactions, and have found that the anharmonicities smear the fusion barrier distributions, somewhat improving the agreement with the experimental 
data. It would be an interesting future problem to extend this treatment to heavy-ion elastic and inelastic scattering, for which the double-folding approach is applicable and thus a fully microscopic approach can be developed using the multi-reference density functional theory. That approach would also be useful in applications to nuclear data as well as the problem of nuclear transmutation [24].

\section{Acknowledgements}

This work was partially supported by the National Natural Science Foundation of China under Grant Nos. 11305134, 11105111, and the Fundamental Research Funds for the Central University (XDJK2013C028).

\section{References}

[1] K. Hagino and N. Takigawa, Prog. Theor. Phys. 128, 1001 (2012); http://www.nucl.phys.tohoku.ac.jp/ hagino/fusion-corr.pdf

[2] M. Dasgupta, D.J. Hinde, N. Rowley and A.M. Stefanini, Annu. Rev. Nucl. Part. Sci. 48, 401 (1998).

[3] A.B. Balantekin and N. Takigawa, Rev. Mod. Phys. 70, 77(1998).

[4] B.B. Back, H. Esbensen, C.L. Jiang and K.E. Rehm, Rev. Mod. Phys. 86, 317 (2014).

[5] L.F. Canto, P.R.S. Gomes, R. Donangelo, J. Lubian and M.S. Hussein, Phys. Rep. 596, 1 (2015).

[6] N. Rowley and K. Hagino, Phys. Rev. C91, 044617 (2015).

[7] K. Hagino and J.M. Yao, Phys. Rev. C91, 064606 (2015).

[8] C.Y. Wong, Phys. Rev. Lett. 31, 766 (1973).

[9] D.L. Hill and J.A. Wheeler, Phys. Rev. 89, 1102 (1953).

[10] D.M. Brink, Semi-Classical Methods for Nucleus-Nucleus Scattering, (University Press, Cambridge, 1985).

[11] N. Poffé, N. Rowley and R. Lindsay, Nucl. Phys. A410, 498 (1983).

[12] K. Hagino, N. Rowley, and A.T. Kruppa, Comp. Phys. Comm. 123, 143 (1999).

[13] N. Rowley, G.R. Satchler, and P.H. Stelson, Phys. Lett. B254, 25 (1991).

[14] J.R. Leigh et al., Phys. Rev. C52, 3151 (1995).

[15] M. Beckerman et al., Phys. Rev. C23, 1581 (1981).

[16] A.M. Stefanini et al., Phys. Rev. Lett. 74, 864 (1995).

[17] J.M. Yao, M. Bender, and P.-H. Heenen, Phys. Rev. C91, 024301 (2015).

[18] M. Bender, P.-H. Heenen, and P.-G. Reinhard, Rev. Mod. Phys. 75, 121 (2003).

[19] J.M. Yao, K. Hagino, Z.P. Li, J. Meng, and P. Ring, Phys. Rev. C89, 054306 (2014).

[20] National Nuclear Data Center, http://www.nndc.bnl.gov/.

[21] J.M. Allmond et al., Phys. Rev. C90, 034309 (2014).

[22] P.W. Zhao, Z.P. Li, J.M. Yao, and J. Meng, Phys. Rev. C82, 054319 (2010).

[23] D. Bourgin et al., Phys. Rev. C90, 044610 (2014).

[24] E.Sh. Sukhovitskii, Y.-O. Lee, J. Chang, S. Chiba, and O. Iwamoto, Phys. Rev. C62, 044605 (2000). 